\section{OP021/\#475 SEPARATING THE BRCA1 AND BRCA2 PHENOTYPE, A PATHWAY ANALYSIS}

${ }^{1} \mathrm{~L}$ Rubinsak*, ${ }^{2} \mathrm{~S} \mathrm{Kim},{ }^{2} \mathrm{H}$ Jang, ${ }^{3} \mathrm{G}$ Mor, ${ }^{3} \mathrm{~S}$ Galoforo, ${ }^{3} \mathrm{H}$ Ramos, ${ }^{2} \mathrm{R}$ Rattan, ${ }^{3} \mathrm{~A}$ Alvero, ${ }^{1} \mathrm{R}$ Gogoi. 'Wayne State University/Karmanos Cancer Institute. Division of Gynecologic Oncology, Gyn Oncology, Detroit, USA; ${ }^{2}$ Wayne State University, Oncology, Detroit, USA; ${ }^{3}$ Wayne State University, Obstetrics and Gynecology, Detroit, USA

\subsection{6/ijgc-2021-IGCS.38}

Objectives To identify gene expression profiles and interacting pathways in BRCA1- and BRCA2- associated high grade serous ovarian cancer (HGSOC) as compared to one another and to BRCA wild type, homologous recombination proficient (HRP) tumors.

Methods of 657 total HGSOC samples, 15 BRCA2 mutated (2.3\%), 16 (2.4\%) BRCA1 mutated, and 375 (57.1\%) HRP samples were analyzed. Gene expression data was collected from Tempus and unpaired t-tests were used to identify differentially expressed genes (DEG) with unadjusted p-value $<0.05$ and fold change of 1.5. Meta and pathway analyses were performed among BRCA1, BRCA2 and HRP groups using Venn diagram and Advaita Bio's iPathwayGuide. BRCA mutated and wild type (wt) ID8 mouse cell lines were used for protein expression and seahorse assay for metabolism analysis.

Results From 18,284 genes with measured expression, 843 (4.6\%) DEG were found between BRCA2 vs BRCA1, 748 (4.1\%) between BRCA2 vs HRP and 1,858 (10.2\%) between BRCA1 and HRP. On meta-analysis of the three comparisons, pathway analysis revealed significant involvement of Wnt signaling pathway and oxidative phosphorylation unique to BRCA2 group compared to fibroblast growth factor signaling and PI3K-Akt signaling for BRCA1. Western blot analysis confirmed higher expression of oxidative phosphorylation complex proteins in BRCA1/BRCA2 mutated lines and differential expression of $\beta$ catenin between BRCA mutated versus wt cell lines. Seahorse assay showed higher oxidative consumption rate in BRCA mutated versus wt cells.

Conclusions Our study identified differential pathway regulation for BRCA2 versus BRCA1 associated HGSOC, suggesting each should be considered a separate phenotype with unique opportunities for targeted therapy.

\section{OP022/\#597 OPTIMIZING THE NUMBER OF CYCLES OF NEOADJUVANT CHEMOTHERAPY IN ADVANCED EPITHELIAL OVARIAN CARCINOMA: A PROPENSITY-SCORE MATCHING ANALYSIS}

${ }^{1} \mathrm{~A}$ Rosati*, ${ }^{1} \mathrm{C}$ Marchetti, ${ }^{2} \mathrm{~F}$ De Felice, ${ }^{1} \mathrm{~S}$ Boccia, ${ }^{1} \mathrm{~L}$ Vertechy, ${ }^{1} \mathrm{M}$ Pavone, ${ }^{1} \mathrm{E}$ Palluzzi, ${ }^{1} \mathrm{G}$ Scambia, ${ }^{1} \mathrm{~A}$ Fagotti. 'Università Cattolica del Sacro Cuore, Department of Woman and Child Health and Public Health, Woman Health Area, Fondazione Policlinico Universitario A. Gemelli Irccs, Roma, Italy; ${ }^{2}$ Policlinico Umberto I, 'Sapienza' University of Rome, Department of Radiotherapy, Rome, Italy

\subsection{6/ijgc-2021-IGCS.39}

Objectives Neoadjuvant chemotherapy and interval debulking surgery are widely offered in advanced ovarian cancer patients; the number of NACT cycles to be given is still an issue. Our aim was to compare survival outcomes of patients with advanced ovarian cancer treated with $<4$ or more NACT cycles.

Methods A cohort of patients with stage III-IV epithelial OC undergoing NACT followed by IDS was identified. Patients were classified in group A $(\leq 4$ cycles) and group B $(>4$
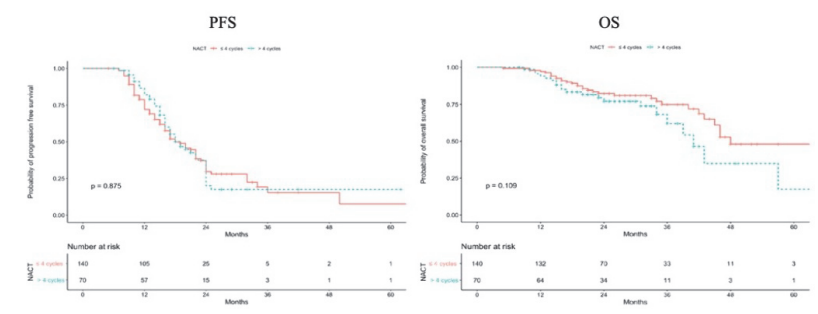

Abstract OP022/\#597 Figure 1 Overall survival and disease free survival in group A and group B

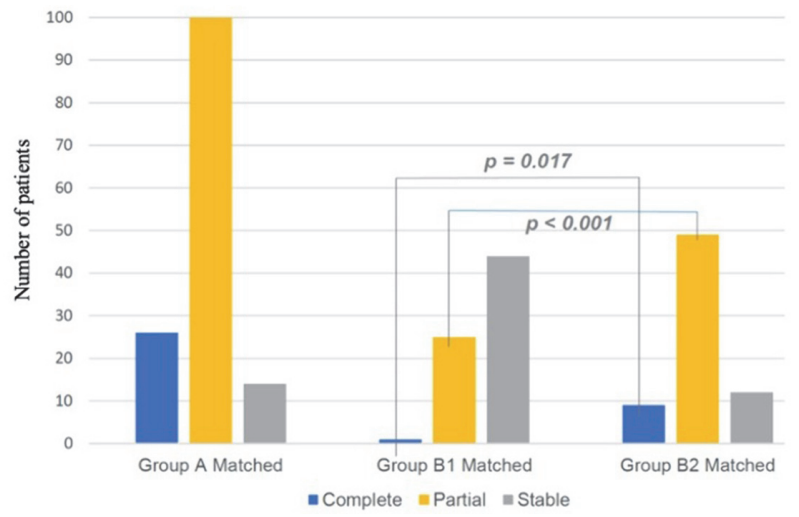

Abstract OP022/\#597 Figure 2 Treatment response between groups. Group A (3-4 cycles); Group B (at least 6 cycles); B1 response assessment after 3-4 cycles; B2 response assessment after at least 6 cycles

cycles). Selection bias was avoided using propensity score matching (2:1 ratio).

Results 140 (group A) and 70 (group B) patients were included. After the propensity score matching, there were no imbalances in baseline characteristics. BRCA status was associated to improved $\mathrm{OS}(\mathrm{HR}=0.41 ; 95 \% \mathrm{CI}$ 0.18.0.92, $\mathrm{p}=0.032)$ and residual tumor to decreased OS $(\mathrm{HR}=1.93$; 95\% CI 1.08 3.46, $\mathrm{p}=0.026)$. Statistically significant differences were not observed in OS (2-year OS $82.4 \%$ for group A versus $77.1 \%$ for group $B, p=0.109$ ) and PFS (2-year PFS 29.7\% for group A versus $20.0 \%$ for group $A, p=0.875$ ) (figure 1). In group $B$, the administration of $>4$ cycles was related to an additional chance of achieving complete (12.9\%) and partial (34.3\%) responses compared to responses after 3-4 cycles (figure 2).

Conclusions Receiving more than 4 cycles of NACT is no detrimental in terms of OS and PFS in advanced ovarian cancer. Response rates can increase following further cycles administration.

\section{OP023/\#658 CORRELATION OF HRD STATUS WITH CLINICAL AND SURVIVAL OUTCOMES IN PATIENTS WITH ADVANCED-STAGE OVARIAN CANCER UNDERGOING FRONTLINE AND MAINTENANCE THERAPY}

${ }^{1} \mathrm{~T}$ Sims ${ }^{*},{ }^{2} \mathrm{~A}$ Sood, ${ }^{2} \mathrm{~S}$ Westin, ${ }^{3} \mathrm{~B}$ Fellman, ${ }^{2} \mathrm{~J}$ Unke, ${ }^{2} \mathrm{~K}$ Rangel, ${ }^{2} \mathrm{~T}$ Hilton, ${ }^{2} \mathrm{~N}$ Fleming. ${ }^{1}$ The University of Texas MD anderson Cancer Center, Gynecologic Oncology and Reproductive Medicine, Houston, USA; ${ }^{2}$ The University of Texas MD anderson Cancer Center, Gynecologic Oncology and Reproductive Medicine, Houston, USA; ${ }^{3}$ The University of Texas MD Anderson Cancer Center, Biostatistics, Houston, USA

10.1136/ijgc-2021-IGCS.40 